\title{
Review of: "High-efficiency broadband achromatic metalens for near-IR biological imaging window"
}

\author{
Tao $\mathrm{Li}^{1}$ \\ 1 Nanjing University
}

Potential competing interests: The author(s) declared that no potential competing interests exist.

In the field of optical imaging, the working efficiency of devices is always the key index in its application. Over the past decade, metasurface as new flat-optics design blooms, showing exciting potential to upgrade the conventional diffractive optics. Among versatile metasurface devices, metalens is undoubtedly arrested the major attention as it is kindly expected to be the compact substitute of conventional imaging module. Nevertheless, it inherits the drawback of large chromatism of diffractive optical elements (DOE). Therefore, tremendous researches have been carried out to correct the chromatic aberration by elaborate meta-designs. Unfortunately, most of these approaches gain the broadband achromatism in sacrifice of efficiency, which severely hinders their practical applications.

Recently, Y. Wang et al. reported their significant progress in achieving high efficient broadband achromatic metalens (Nature Communications 12, 5560 (2021)). By adopting a CMOS compatible etching process technology, they successfully fabricated TiO2 metalens with record-high aspect ratio of nano-pillar ( $>=37.5$ ) with a relatively high height of $1.5 \mathrm{um}$. It convincingly gives rise to a high average efficiency (77\%-88.5\%) over a broad achromatic wavelength range of 650-1000 nm (i.e., the first biological imaging window). The subsequent upconversion imaging results based on this metalens further show comparable image qualities with that from a commercial achromatic objective. Although this upconversion imaging at certain wavelength $(655 \mathrm{~nm}$ ) with only $20 \mathrm{~nm}$ linewidth may not the best examiner for such an achromatic metalens, the high image quality definitely shows its competitive applications in bio-imaging. Undoubtedly, this high-efficiency metalens provides people great encouragement in developing practical devices.

In fact, several recent papers have pointed out that the achromatic metalens has an intrinsic constraint among its efficiency, bandwidth, numerical aperture (NA), and lens size. Almost all of metalenses in past works including the current one, have to sacrifice one to several aspects to improve its efficiency, no matter what kinds of nano-units designed. Because the chromatic dispersion compensation in a large scale can only be fulfilled by propagation phase, which ultimately relies on the height of nano-pillars. Thus, the overall improvement in efficiency only depends on the thickness of metalens. Indeed, this reported high efficiency metalens has very limited size with a diameter of $25 \mathrm{um}$, though it has a thickness of $1.5 \mathrm{um}$. By theoretical estimation, for a macroscopic large flat-lens (e.g., $\mathrm{cm}$ or $\mathrm{mm}$ scale), its thickness needs to be 
the order of tens or hundreds micrometers without great decreases in its efficiency and NA. It is almost impossible based on recent fabrication technique. Another comment is the necessarity of achromatic metalens as being used for the bio-imaging. Since the manuscript does provide a clear scheme of microscope system, it would be questioned whether the utility of metalens can simplify the imaging framework. If the metalens only acts a substitute of objective lens without change the whole device system (e.g., imaging distance, etc.), the advantage of its ultra-thin and ultra-light would be very limited. From these two points, I think we still need to be cautious about the real applications of metalens in near future besides this exciting progress. 